\title{
MicroRNA-27a functions as an oncogene in human osteosarcoma by targeting CCNG1
}

\author{
TAO LIN ${ }^{1}$, QUANPING MA ${ }^{2}$, YUEFENG ZHANG ${ }^{3}$, HONGFEI ZHANG $^{4}$, JIAPENG YAN $^{4}$ and CHANGHONG GAO ${ }^{1}$ \\ ${ }^{1}$ Department of Orthopedics, Jinan Central Hospital Affiliated to Shandong University; ${ }^{2}$ Clinical Laboratory, \\ Jinan Fourth People's Hospital; ${ }^{3}$ Major Surgery, Shandong Yellow River Hospital, Jinan, Shandong 250000; \\ ${ }^{4}$ Department of Orthopedics, Affiliated Hospital of Weifang Medical University, Weifang, Shandong 261031, P.R. China
}

Received July 12, 2017; Accepted October 24, 2017

DOI: $10.3892 / \mathrm{ol} .2017 .7389$

\begin{abstract}
Osteosarcoma is the most common type of malignant tumor arising from bone in children and adolescents. Accumulating evidences have shown the aberrant expression of numerous miRNAs is associated with the development and metastasis of osteosarcoma. The present study was conducted to investigate miR-27a expression in osteosarcoma tissues and cells. In the present study, quantitative RT-qPCR was used to measure the expression levels of miRNA and mRNA in osteosarcoma tissues and cells. Transwell assays were used to detect the effects of miR-27a on the invasive and migratory potential of cells. Luciferase reporter and western blot analysis were conducted to confirm cyclin G1 (CCNG1) as the target gene of miR-27a. The results showed that miR-27a was significantly upregulated in human osteosarcoma tissues and cell lines. The western blot analysis revealed that the overexpression of miR-27a suppressed CCNG1 protein expression. Luciferase reporter assays confirmed that CCNG1 is a direct target of miR-27a in osteosarcoma cells. The results suggest that miR-27a downregulates CCNG1 expression in osteosarcoma and acts as an oncogene directly targeting CCNG1. Thus, the miR-27a/CCNGI axis is a potential therapeutic target for human osteosarcoma.
\end{abstract}

\section{Introduction}

Osteosarcoma is the most common type of malignant tumor arising from bone in children and adolescents, with high biologic aggressiveness and comprising $2.4 \%$ of all malignancies in pediatric patients $(1,2)$. Although modern treatment methods combine surgery, multiagent chemotherapy, and sometimes radiotherapy, the 5-year survival rate for patients diagnosed with osteosarcoma is $60-70 \%$ (3). The past decade

Correspondence to: Dr Changhong Gao, Department of Orthopedics, Jinan Central Hospital Affiliated to Shandong University, 105 Jiefang Road, Jinan, Shandong 250000, P.R. China E-mail: ucj221@163.com

Key words: microRNA-27a, osteosarcoma, cyclin G1, migration, invasion has revealed the molecular pathogenesis of osteosarcoma expands, and that potential therapeutic targets are being identified $(4,5)$. It is essential to comprehensively understand molecular mechanisms in order to improve the prognosis of patients with osteosarcoma through tumour-targeted therapies.

MicroRNAs (miRNAs) are a class of non-coding, small regulatory RNAs, 21-23 nucleotides in length (6), transcribed from introns or non-protein-coding genes, which cleavage of their target mRNAs by binding to complementary sites in their 3'-untranslated regions (UTR) or mediate translational suppression (7). miRNAs play crucial roles in cell cycle regulation, differentiation, apoptosis, tumorigenesis, migration and invasion (8-10). Accumulating evidences have shown the aberrant expression of numerous miRNAs is associated with the development and metastasis of cancer $(11,12)$, including human osteosarcoma (13). Previous studies have researched the role of miRNAs in osteosarcoma using miRNA profile $(14,15)$. Pan et al and Salah et al have reported that miR-27a promoted the proliferation, migration and invasion of human osteosarcoma cells $(16,17)$. However, the miR-27a expression pattern and clinical value in human osteosarcoma remain to be determined.

Cyclin G1 (CCNG1) was primarily identified as a novel member of the cyclin family with homology to c-src (18), and importantly, it was first identified as a p53-regulated transcript induced by DNA damage (19). CCNG1 is transcriptionally activated by $\mathrm{p} 53$, and negatively regulates p 53 family proteins. CCNG1, a master tumor suppressor, is directly regulated by miR-27b/miR-508-5p (20), miR122 (21), and miR1271 (22).

In the present study, we investigated whether miR-27a expression in osteosarcoma tissues and cells was upregulated, as compared to normal non-cancer tissue and a normal human osteoblastic cell line. miR-27a significantly promoted osteosarcoma cell migration and invasion in vitro. Additionally, CCNG1 was identified as a direct target of miR-27a. miR-27a/CCNG1 axis is a potential therapeutic target for human osteosarcoma.

\section{Materials and methods}

Tissue samples. A total of 48 paired primary osteosarcoma and their matched adjacent non-cancerous bone tissue samples were collected from patients who underwent surgery at the Department of Orthopedics, Jinan Central Hospital Affiliated 
to Shandong University (Jinan, China), between 2013 and 2016. The patients had not received chemotherapy prior to surgery. All the tissue samples were instantly snap-frozen in liquid nitrogen at the time of surgery, and stored at $-80^{\circ} \mathrm{C}$ for using in the subsequent tests. The diagnosis of osteosarcoma was confirmed pathologically.

Ethics approval. The study was approved by the Research Ethics Committee of Jinan Central Hospital Affiliated to Shandong University, and informed consent was obtained from all the patients. Procedures performed in the studies involving human participants were in accordance with the ethical standards of the Institutional and/or National Research Committee and with the 1964 Helsinki Declaration and its later amendments or comparable ethical standards.

Cell culture and transfection. Four osteosarcoma cell lines (HOS, SaOS2, 143B and MG63), and the osteoblastic cell line (hFOB1.19) were obtained from the Type Culture Collection of the Chinese Academy of Sciences (Shanghai, China). Cells were cultured in RPMI-1640 (Gibco; Thermo Fisher Scientific, Inc., Waltham, MA, USA) supplemented with $10 \%$ fetal bovine serum (FBS), $100 \mathrm{mg} / \mathrm{ml}$ streptomycin and $100 \mathrm{U} / \mathrm{ml}$ penicillin (Invitrogen; Thermo Fisher Scientific, Inc., Waltham, MA, USA) at $37^{\circ} \mathrm{C}$ in a humidified incubator containing $5 \% \mathrm{CO}_{2}$. The cells were transfected with the indicated nucleotides or plasmid using Lipofectamine 2000 reagent (Invitrogen; Thermo Fisher Scientific, Inc.) according to the manufacturer's protocol.

$R N A$ extraction and reverse transcription-quantitative $P C R$ $(R T-q P C R)$. Total RNAs were extracted from the cultured tissue samples and cells using TRIzol reagent (Invitrogen; Thermo Fisher Scientific, Inc.) according to the manufacturer's protocol. miRNA cDNA was synthesized using the One-Step PrimeScript miRNA cDNA synthesis kit (Takara Biotechnology Co., Ltd., Dalian, China). RT-qPCR was performed with SYBR-Green Premix Ex Taq II (Takara Biotechnology Co., Ltd.) with a StepOne-Plus real-time PCR system (Applied Biosystems; Thermo Fisher Scientific, Inc., Waltham, MA, USA). The level of mature miR-27a was normalized relative to U6 endogenous control and CCNG1 expression was normalized relative to $\beta$-actin (endogenous control) using the $2^{-\Delta \Delta \mathrm{Cq}}$ method and at least 3 independent experiments were performed to generate each data set. Primers used were purchased from Guangzhou RiboBio Co., Ltd. (Guangzhou, China).

Cell transwell assay. Cell transwell assay was performed in a 24-well plate with $8 \mathrm{~mm}$ pore size chamber inserts (Corning Incorporated, Corning, NY, USA). For the migration and invasion assays, after transfection with either the mimic/inhibitor or control $\mathrm{miR}, 1 \times 10^{5}$ cells/well were placed into the upper chamber with or without membrane Matrigel (BD Biosciences, Franklin Lakes, San Jose, CA, USA), respectively. In transwell assay, the upper chamber contained $200 \mu \mathrm{l}$ serum-free medium, and the lower chambers contained $800 \mu \mathrm{l}$ of medium with $15 \% \mathrm{FBS}$. After $36 \mathrm{~h}$ of incubation at $37^{\circ} \mathrm{C}$ in $5 \% \mathrm{CO}_{2}$, the cells on the upper surface of the membrane were removed, and the cells that had moved to the bottom of the chamber were fixed with $100 \%$ methanol for $30 \mathrm{~min}$ and stained with $0.1 \%$ crystal violet for $30 \mathrm{~min}$. The stained cells were imaged and counted using an inverted microscope (Olympus Corporation, Tokyo, Japan).

Western blot analysis. Cells were lysed in RIPA buffer. The proteins were extracted and separated by $10 \%$ sodium dodecyl sulphate-polyacrylamide gel electrophoresis. The separated proteins were electro-transferred to polyvinylidene difluoride membrane (Millipore, Boston, MA, USA). The membrane was blocked with 5\% non-fat milk and incubated overnight with primary anti-AEG-1 (Invitrogen; Thermo Fisher Scientific, Inc.) at $4^{\circ} \mathrm{C}$. The membranes were incubated for $1 \mathrm{~h}$ at $25^{\circ} \mathrm{C}$ with horseradish peroxidase-conjugated secondary antibodies (1:2,000; Bosis, Beijing, China). Relative protein expression was obtained by normalization against glyceraldehyde 3-phosphate dehydrogenase. The proteins of interest were revealed by the ECL western blotting kit (PSC Biotech Pte., Ltd., Shanghai, China). Densitometry analysis of the protein blots was performed by Lab Works ${ }^{\mathrm{TM}}$ software (UVP, Inc., Upland, CA, USA).

Dual-luciferase reporter assay. For dual-luciferase reporter assays, the 3'-UTR of CCNG1 containing miR-27a binding sites were cloned into a pmirGLO dual-luciferase vector (Promega Corporation, Madison, WI, USA) to generate wild-type (WT) pmirGLO-CCNG1-1 3'-UTR. The mutant (MUT) 3'-UTR of CCNG1 gene with miR-27a target sites were generated using a Site-Directed Mutagenesis kit (Agilent Technologies, Inc., Santa Clara, CA, USA), and cloned into a pmirGLO dual-luciferase vector (Promega Corporation) to generate MUT pmirGLOCCNG1-1 3'-UTR. The WT pmirGLO-CCNG1-1 3'-UTR and MUT pmirGLO-CCNG1-1 3'-UTR were co-transfected with miR-27a mimics, inhibitor or negative control (NC) by using Lipofectamine 2000 (Invitrogen; Thermo Fisher Scientific, Inc.). After incubation for two days, luciferase activity was detected by using a dual-Glo luciferase assay system (Promega Corporation) according to the manufacturer's protocol.

Statistical analysis. The results are reported as mean $\pm \mathrm{SD}$ and data analysis was performed using SPSS 17.0 software (SPSS, Inc., Chicago, IL, USA). The relationship between the expression of miR-27a and CCNG1 were confirmed by Spearman's rho correlation. Differences between the two groups were calculated using the Student's t-test or Chi-square test. $\mathrm{P}<0.05$ was considered to indicate a statistically significant difference.

\section{Results}

miR-27a expression is upregulated and inversely correlates with CCNG1 in human osteosarcoma tissues and cells. To investigate the expression and significance of miR-27a in human osteosarcoma, the study first evaluated miR-27a expression in 48 paired primary osteosarcoma and their matched adjacent non-cancerous bone tissue samples using RT-qPCR. miR-27a expression was clearly upregulated in osteosarcoma tissues compared with non-cancerous bone tissue samples (Fig. 1A). Furthermore, miR-27a was also significantly increased in the four osteosarcoma cell lines (HOS, SaOS2, 143B and MG63) compared with that of the osteoblastic cell line hFOB1.19 (Fig. 1B). 
A
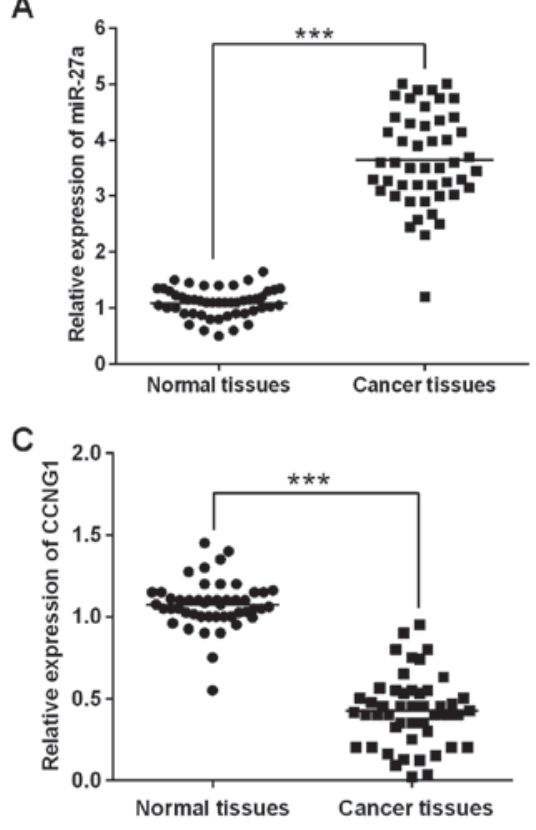

B

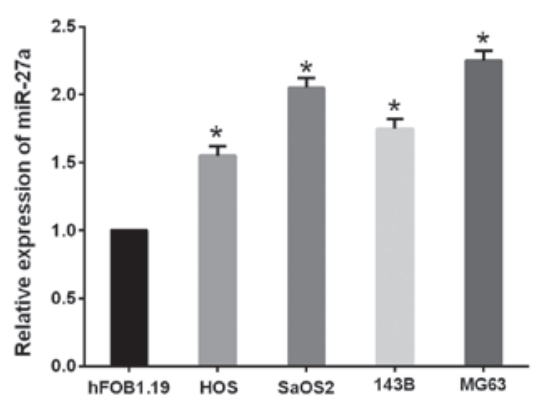

D

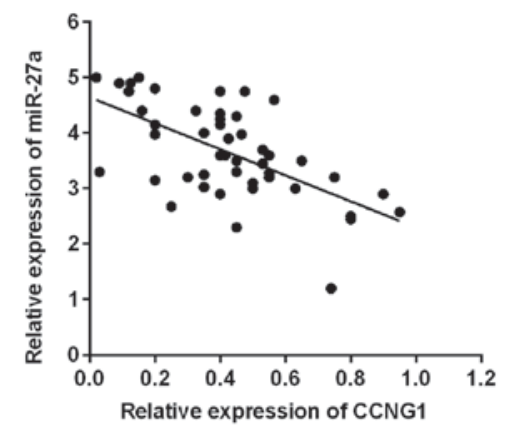

Figure 1. miR-27a expression is upregulated and inversely correlates with CCNG1 in human osteosarcoma tissues and cells (A and C). miR-27a and CCNG1 expression was detected in 48 pairs of osteosarcoma tissues compared with their matched adjacent non-cancerous bone tissue samples. (B) miR-27a in osteosarcoma and osteoblastic cell line were measured by RT-qPCR $(\mathrm{P}<0.05)$. (D) Spearman's rho analysis of miR-27a expression and CCNG1 in 48 osteosarcoma tissues. ${ }^{*} \mathrm{P}<0.05,{ }^{* * *} \mathrm{P}<0.001 ; \mathrm{CCNG1}$, cyclin G1; RT-qPCR, reverse transcriptase quantitative PCR.

The study further investigated the expression of CCNG1 in 48 pairs of osteosarcoma tissue and adjacent normal tissues. The results showed that CCNG1 was significantly downregulated in osteosarcoma tissue compared with the paired-adjacent normal tissues and inversely correlated with the miR-27a level in cervical cancer tissues (Fig. 1C and D), suggesting that miR-27a may have a critical role as an oncogene in human osteosarcoma. However, the specific function of miR-27a in human osteosarcoma remained to be determined.

Effect of miR-27a on human osteosarcoma cell migration and invasion. To reveal the biological effects of miR-27a on the migration and invasion of human osteosarcoma cells, cell migration and invasion abilities were determined in the 143B and HOS cells transfected with the miR-27a mimic, miR-27a inhibitor, or NC by transwell assays. The re-expression levels of miR-27a in transfected and normal cells were detected by RT-qPCR. It was found that overexpression of miR-27a significantly promoted the migration and invasion capacities in the 143B cells and HOS cells (Fig. 2A). Similarly, Fig. 2B shows the re-expression levels of miR-27a in 143B and HOS cells transfected with the miR-27a mimic or inhibitor compared with corresponding NC. These results suggested that miR-27a acts as an oncomiR and promotes cell migration and invasion during osteosarcoma progression.

$C C N G 1$ as a direct binding target of $m i R-27 a$. To determine the mechanisms underlying how miR-27a functions as an oncogene of human osteosarcoma pathology, we used the bioinformatics algorithms TargetScan to search for the potential target genes of miR-27a. Our analysis revealed that CCNG1 was a potential target of miR-27a based on putative target sequences at position 260-267 bp of the CCNG1 3'UTR (Fig. 3A). To confirm CCNG1 as a direct target of miR-27a, we engineered luciferase reporter constructs, containing the WT or MUT 3'UTR of the CCNG1 gene. Luciferase reporter assay showed that miR-27a significantly decreased the luciferase activity of the CCNG1 3'UTR but not that of the MUT in 143B cells (Fig. 3B). We further examined whether CCNG1 levels are negatively regulated by miR-27a in osteosarcoma cell lines. As shown in Fig. 3C and D, mRNA and protein levels of CCNG1 were significantly enhanced or reduced in response to $\mathrm{miR}-27 \mathrm{a}$ mimics/inhibitor in HOS cells, compared to the corresponding controls. Taken together, these data strongly suggest that CCNG1 is a direct target of miR-27a in human osteosarcoma.

\section{Discussion}

Osteosarcoma is the most common type of malignant tumor arising from bone in children and adolescents that threatens human life. The past decade has revealed the developement of molecular pathogenesis of osteosarcoma; thus, there is a search for potential therapeutic targets $(4,5)$. miRNAs are a type of small-molecule non-coding RNA, that play vital roles in regulating expression levels of post-transcriptional gene. The functions of miRNAs vary in different clinical diseases and may regulate all aspects of bioactivities, including differentiation and development, proliferation, metabolism, viral infection, apoptotic cell death and tumorigenesis (23). Increasing evidence has shown the abnormal expression of miRNAs is associated with the development and metastasis of human osteosarcoma (13). Examination of the relationships between miRNAs and human osteosarcoma possibly provide a new orientation for the diagnosis and therapy of osteosarcoma. In previous studies, partial miRNAs were reported to inhibit osteosarcoma tumorigenicity and to suppress cell proliferation; 
A

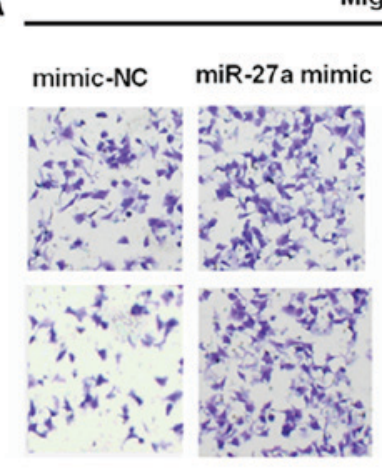

Migration

inhibitor-NC miR-27a inhibitor
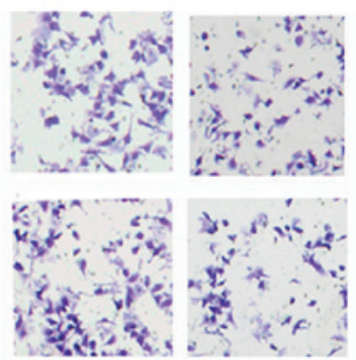

Invasion

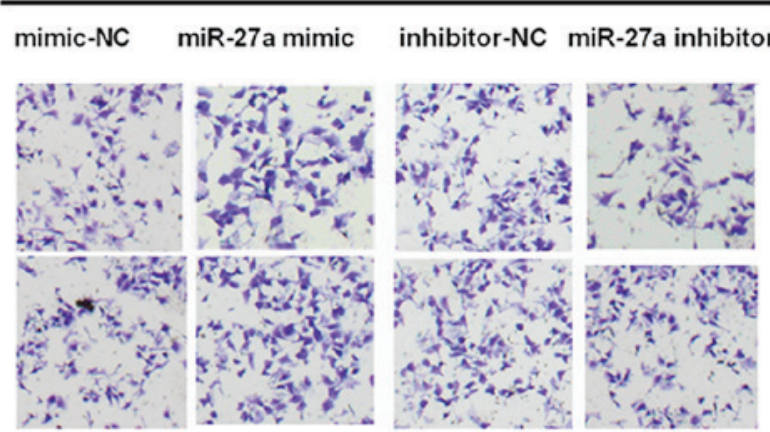

B
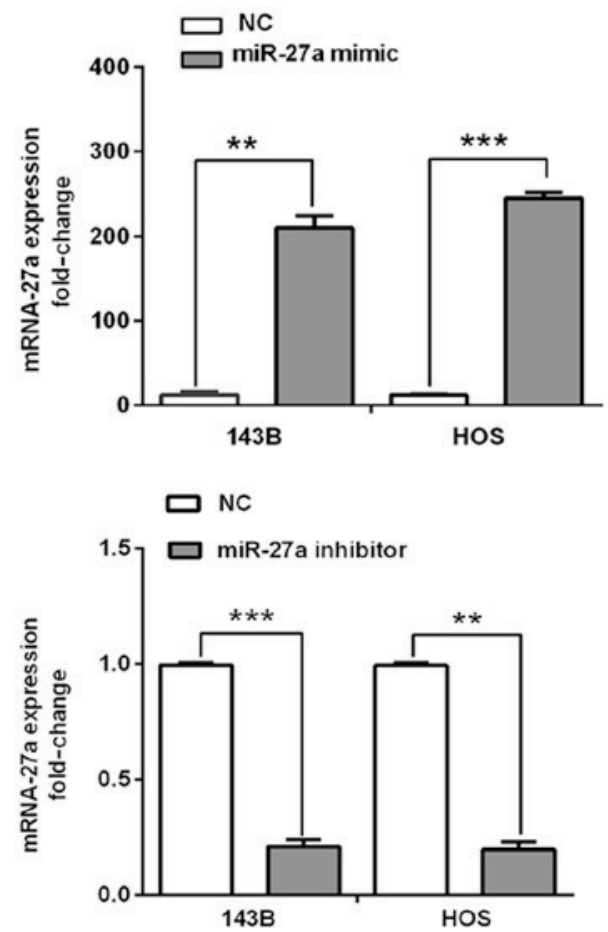

Figure 2. Effect of miR-27a on human osteosarcoma cell migration and invasion. (A) miR-27a promotes the invasion and migration of HOS and 143B cells. (B) miR-27a was re-expressed in the miR-27a mimic/inhibitor transfected HOS and 143B cells. miR-27a levels were determined by RT-qPCR, miR-27a/U6 was calculated as the fold change relative to the negative control. ${ }^{* * *} \mathrm{P}<0.001,{ }^{* *} \mathrm{P}<0.01$; RT-qPCR, reverse transcriptase-quantitative PCR.

A

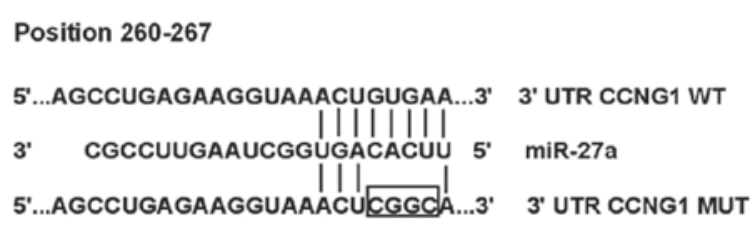

B

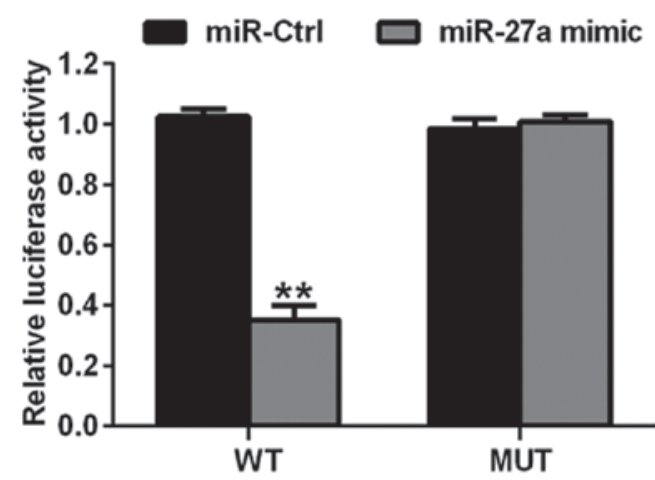

C
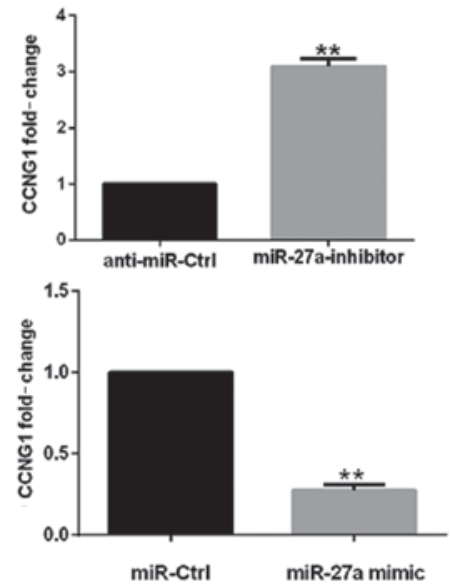

D

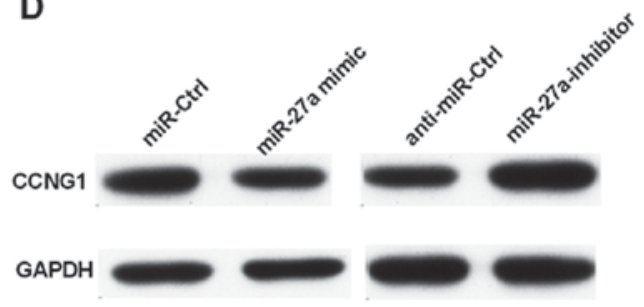

Figure 3. miR-27a negatively regulates CCNG1 in osteosarcoma cells. (A) Specific locations of the binding sites of miR-27a in the wild- and mutant-type 3'UTR-CCNG1. (B) Luciferase reporter assay showed the effect of miR-27a on CCNG1 3'UTR luciferase activity in 143B cells. (C and D) The expression levels of CCNG1 protein in HOS cells transfected with miR-27a mimics and miR-27a inhibitor by western blot analysis. ${ }^{* *} \mathrm{P}<0.01$.

and partially were reported to promote osteosarcoma cell proliferation and induce cell survival (24-26).
Previous studies reported miR-27a functions differentially in various cancer types, including oral squamous cell 
carcinoma (27), breast (28), gastric (29), colorectal (30), and colonic cancer (31). Furthermore, Pan et al have reported that miR-27a could promote proliferation, migration and invasion by targeting MAP2K4 in human osteosarcoma cells (16). Salah et al reported miR-27a contributes to the metastatic properties of osteosarcoma cells (17). CCNG1 was primarily identified as a novel member of cyclin family with homology to c-src (18). CCNG1, a master tumor suppressor, has been shown to be directly regulated by various miRNAs in different tumors (20-22). In a previous study it was reported that CCNG1 inhibited osteosarcoma tumor growth in nude mice (32).

Our results have found that miR-27a acts as an oncogene in human osteosarcoma. The results showed miR-27a expression was upregulated in human osteosarcoma tissues and cells, which is similar to those obtained in previous studies $(14,16)$. The overexpression of miR-27a significantly promoted the migration and invasion capacities in the human osteosarcoma cells. Moreover, the study demonstrated that CCNG1 was a directed target, and that miR-27a expression inversely correlated with CCNG1 in human osteosarcoma tissues and cells. The western blot analysis and Luciferase reporter assay also showed that CCNG1 was regulated by miR-27a.

In conclusion, results of the present study have shown that miR-27a is significantly upregulated and inversely correlates with CCNG1 in osteosarcoma. These results demonstrate that miR-27a has powerful oncogenic, metastatic and invasive regulatory effects that are mediated by CCNG1. The study indicates that miR-27a acts as an oncogene and is a promising therapeutic target in human osteosarcoma.

\section{References}

1. Geller DS and Gorlick R: Osteosarcoma: A review of diagnosis, management, and treatment strategies. Clin Adv Hematol Oncol 8: 705-718, 2010.

2. Raymond AK and Jaffe N: Osteosarcoma multidisciplinary approach to the management from the pathologist's perspective. Cancer Treat Res 152: 63-84, 2009.

3. Ferrari S, Mercuri M, Bacci G, Bielack SS and Jürgens H: Comment on 'Prognostic factors in high-grade osteosarcoma of the extremities or trunk: An analysis of 1,702 patients treated on neoadjuvant Cooperative Osteosarcoma Study Group protocols'. J Clin Oncol 20: 2910-2911, 2002.

4. Broadhead ML, Clark JC, Myers DE, Dass CR and Choong PF: The molecular pathogenesis of osteosarcoma: A review. Sarcoma 2011: 959248, 2011.

5. He JP, Hao Y, Wang XL, Yang XJ, Shao JF, Guo FJ and Feng JX: Review of the molecular pathogenesis of osteosarcoma. Asian Pac J Cancer Prev 15: 5967-5976, 2014.

6. Fang Z, Du R, Edwards A, Flemington EK and Zhang K: The sequence structures of human microRNA molecules and their implications. PLoS One 8: e54215, 2013.

7. He L and Hannon GJ: MicroRNAs: Small RNAs with a big role in gene regulation. Nat Rev Genet 5: 522-531, 2004.

8. Nicoloso MS, Spizzo R, Shimizu M, Rossi S and Calin GA: MicroRNAs - the micro steering wheel of tumour metastases. Nat Rev Cancer 9: 293-302, 2009.

9. Bartel DP: MicroRNAs: Genomics, biogenesis, mechanism, and function. Cell 116: 281-297, 2004.

10. Bueno MJ, Pérez de Castro I and Malumbres M: Control of cell proliferation pathways by microRNAs. Cell Cycle 7 : 3143-3148, 2008.

11. Calin GA and Croce CM: MicroRNA signatures in human cancers. Nat Rev Cancer 6: 857-866, 2006.

12. Saumet A and Lecellier CH: microRNAs and personalized medicine: Evaluating their potential as cancer biomarkers. Adv Exp Med Biol 888: 5-15, 2015.

13. Nugent M: microRNA and bone cancer. Adv Exp Med Biol 889: 201-230, 2015.
14. Jones KB, Salah Z, Del Mare S, Galasso M, Gaudio E, Nuovo GJ, Lovat F, LeBlanc K, Palatini J, Randall RL, et al: miRNA signatures associate with pathogenesis and progression of osteosarcoma. Cancer Res 72: 1865-1877, 2012

15. Gougelet A, Pissaloux D, Besse A, Perez J, Duc A, Dutour A, Blay JY and Alberti L: Micro-RNA profiles in osteosarcoma as a predictive tool for ifosfamide response. Int J Cancer 129: 680-690, 2011.

16. Pan W, Wang H, Jianwei R and Ye Z: MicroRNA-27a promotes proliferation, migration and invasion by targeting MAP2K4 in human osteosarcoma cells. Cell Physiol Biochem 33: 402-412, 2014

17. Salah Z, Arafeh R, Maximov V, Galasso M, Khawaled S, Abou-Sharieha S, Volinia S, Jones KB, Croce CM and Aqeilan RI: miR-27a and miR-27a* contribute to metastatic properties of osteosarcoma cells. Oncotarget 6: 4920-4935, 2015.

18. Tamura K, Kanaoka Y, Jinno S, Nagata A, Ogiso Y, Shimizu K, Hayakawa T, Nojima $\mathrm{H}$ and Okayama H: Cyclin G: A new mammalian cyclin with homology to fission yeast Cig1. Oncogene 8: 2113-2118, 1993

19. Okamoto K and Beach D: Cyclin G is a transcriptional target of the p53 tumor suppressor protein. EMBO J 13: 4816$4822,1994$.

20. Shang Y, Feng B, Zhou L, Ren G, Zhang Z, Fan X, Sun Y, Luo G, Liang J, Wu K, et al: The miR27b-CCNG1-P53-miR-508-5p axis regulates multidrug resistance of gastric cancer. Oncotarget 7: 538-549, 2016.

21. Fornari F, Gramantieri L, Giovannini C, Veronese A, Ferracin M, Sabbioni S, Calin GA, Grazi GL, Croce CM, Tavolari S, et al: MiR-122/cyclin G1 interaction modulates p53 activity and affects doxorubicin sensitivity of human hepatocarcinoma cells. Cancer Res 69: 5761-5767, 2009.

22. Liu X, Ma L, Rao Q, Mao Y, Xin Y, Xu H, Li C and Wang X: MiR-1271 inhibits ovarian cancer growth by targeting cyclin G1. Med Sci Monit 21: 3152-3158, 2015.

23. Huang Y, Shen XJ, Zou Q, Wang SP, Tang SM and Zhang GZ: Biological functions of microRNAs: A review. J Physiol Biochem 67: 129-139, 2011

24. Kushlinskii NE, Fridman MV and Braga EA: Molecular mechanisms and microRNAs in osteosarcoma pathogenesis. Biochemistry (Mosc) 81: 315-328, 2016

25. Ram Kumar RM, Boro A and Fuchs B: Involvement and clinical aspects of microRNA in osteosarcoma. Int J Mol Sci 17: E877, 2016.

26. Ma C, Zhan C, Yuan H, Cui Y and Zhang Z: MicroRNA-603 functions as an oncogene by suppressing $\mathrm{BRCC} 2$ protein translation in osteosarcoma. Oncol Rep 35: 3257-3264, 2016.

27. Zeng G, Xun W, Wei K, Yang Y and Shen H: MicroRNA-27a-3p regulates epithelial to mesenchymal transition via targeting YAP1 in oral squamous cell carcinoma cells. Oncol Rep 36: 1475-1482, 2016

28. Morales S, Gulppi F, Gonzalez-Hormazabal P, FernandezRamires R, Bravo T, Reyes JM, Gomez F, Waugh E and Jara L: Association of single nucleotide polymorphisms in Pre-miR-27a, Pre-miR-196a2, Pre-miR-423, miR-608 and Pre-miR-618 with breast cancer susceptibility in a South American population. BMC Genet 17: 109, 2016.

29. Danza K, Silvestris N, Simone G, Signorile M, Saragoni L, Brunetti O, Monti M, Mazzotta A, De Summa S, Mangia A, et al: Role of miR-27a, miR-181a and miR-20b in gastric cancer hypoxia-induced chemoresistance. Cancer Biol Ther 17: 400-406, 2016.

30. Liu F, Dear K, Huang L, Liu L, Shi Y, Nie S, Liu Y, Lu Y and Xiang H: Association between microRNA-27a rs895819 polymorphism and risk of colorectal cancer: A meta-analysis. Cancer Genet 209: 388-394, 2016.

31. Gao Y, Li BD and Liu YG: Effect of miR27a on proliferation and invasion in colonic cancer cells. Asian Pac J Cancer Prev 14: 4675-4678, 2013.

32. Chen DS, Zhu NL, Hung G, Skotzko MJ, Hinton DR, Tolo V, Hall FL, Anderson WF and Gordon EM: Retroviral vector-mediated transfer of an antisense cyclin G1 construct inhibits osteosarcoma tumor growth in nude mice. Hum Gene Ther 8: 1667-1674, 1997.

This work is licensed under a Creative Commons Attribution-NonCommercial-NoDerivatives 4.0 International (CC BY-NC-ND 4.0) License. 